UWThPh-1996-17

October 22, 2018

\title{
Real Oscillations of Virtual Neutrinos
}

\author{
W. Grimus and P. Stockinger \\ Institut für Theoretische Physik \\ Universität Wien \\ Boltzmanngasse 5 \\ A-1090 Vienna, Austria
}

\begin{abstract}
We study the conditions for neutrino oscillations in a field theoretical approach by taking into account that only the neutrino production and detection processes, which are localized in space around the coordinates $\vec{x}_{P}$ and $\vec{x}_{D}$, respectively, can be manipulated. In this sense the neutrinos whose oscillations are investigated appear as virtual lines connecting production with detection in the total Feynman graph and all neutrino fields or states to be found in the discussion are mass eigenfields or eigenstates. We perform a thorough examination of the integral over the spatial components of the inner neutrino momentum and show that in the asymptotic limit $L=\left|\vec{x}_{D}-\vec{x}_{P}\right| \rightarrow \infty$ the virtual neutrinos become "real" and under certain conditions the usual picture of neutrino oscillations emerges without ambiguities.
\end{abstract}




\section{Introduction}

In particle physics, oscillation phenomena have so far only been observed in the $K^{0} \bar{K}^{0}$ and $B^{0} \bar{B}^{0}$ systems. Analogous oscillations are expected to take place between neutrinos of different lepton flavours [1] if neutrinos are massive particles and if individual lepton numbers are not conserved. This non-conservation manifests itself in a neutrino mass matrix which is non-diagonal in the weak basis, i.e. the basis where the mass matrix of the charged leptons is diagonal and the charged current interactions of the standard model $(\mathrm{SM})$ is of the form

$$
\mathcal{L}_{C C}=-\frac{g}{\sqrt{2}}\left(\sum_{\ell=e, \mu, \tau} \bar{\nu}_{\ell L} \gamma^{\mu} \ell_{L} W_{\mu}^{+}+\text {h.c. }\right) .
$$

Though neutrino masses and mixings rank among physics beyond the SM it is nevertheless reasonable to assume that neutrino production and detection are within the province of the SM interactions. In this sense the left-handed neutrino fields in eq. (1) are linear combinations of mass eigenfields, i.e.

$$
\nu_{\ell L}(x)=U_{\ell j} \nu_{j L}(x)
$$

with a unitary $3 \times 3$ matrix $U$ and neutrino fields $\nu_{j}$ with mass $m_{j}$.

The standard approach to neutrino oscillations (see, e.g., ref. [2]) is important for its simplicity and its physical insight but is not sufficient for a complete understanding. (See, e.g., ref. [3] for a list of its conceptual problems.) It is well known that it works only in the extreme relativistic limit. Wave-packet treatments [4] are not totally satisfactory either without knowing size and form of the wave packets. However, it is clear that, if the particles involved in the neutrino production process are assumed to have definite 4-momenta, the neutrino is forced to have a definite 4-momentum too and hence is in one of the mass eigenstates. Therefore, in order to observe neutrino oscillations one needs a sufficient spread of momentum (or energy) of the particles in the production and detection mechanism. It has been stressed in the literature [3, 5] that the correct way to avoid all difficulties and ambiguities associated with neutrino oscillations is to concentrate on those things which can really be manipulated or observed like the particles responsible for neutrino production and the target responsible for neutrino detection. This amounts to including the production and detection mechanisms localized at the coordinates $\vec{x}_{P}$ and $\vec{x}_{D}$, respectively, in the calculation such that the neutrinos propagating from $\vec{x}_{P}$ to $\vec{x}_{D}$ are virtual particles [6] or intermediate states (see ref. [5] for a field-theoretical treatment and ref. [3] for a quantum-mechanical treatment along these lines).

In this paper we choose the field-theoretical approach and apply it to the antineutrino production as it occurs in a reactor and assume that the antineutrinos are detected by elastic electron scattering. (This is, e.g., the situation in the MUNU experiment [7].) The

antineutrino flux is produced by the $\beta$-decay of the fission products in the reactor. We simplify this mechanism by taking only the $\beta$-decay of the neutron and assuming that both neutron and proton are bound to nuclei at rest. This merely serves to keep our 
notation as simple as possible and has no impact on the essence of our discussion. The main points in our treatment of neutrino oscillations are the following:

- The neutron and the proton are bound to nuclei in stationary states localized at the coordinates $\vec{x}_{P}$ whereas the target electron is bound in an atom at the point $\vec{x}_{D}$. Neutrino production and detection are macroscopically separated by a distance $L \equiv|\vec{L}|$ with $\vec{L} \equiv \vec{x}_{D}-\vec{x}_{P}$.

- All particles in the final state are described by plane waves. This is justified for the electron in the detector and all the more for the other electron from the $\beta$-decay and the antineutrino which are both not observed. In the event rate it has to be summed (integrated) over a complete set of states for these unobserved particles and plane waves obviously serve this purpose. (This is one of the points which distinguishes our work from ref. [5].)

- The internal (anti)neutrino is represented by its Feynman propagator. The integration over this internal line with momentum $q$ is coupled to the macroscopic distance between neutrino production and detection by $\int d^{3} q \exp (-i \vec{q} \cdot \vec{L})\left(q^{2}-m_{j}^{2}+i \epsilon\right)^{-1} \ldots$ for a neutrino with mass $m_{j}$. We use a mathematical theorem which is proved in the appendix to obtain the leading term of the amplitude in the limit $L \rightarrow \infty$. We will see that in this asymptotic limit the antineutrino is "forced" to be on its mass shell and the momentum $\vec{q}$ is aligned with $-\vec{L}$.

\section{The oscillation amplitude}

We consider the following weak process

$$
\begin{array}{lll}
n \rightarrow p+e^{-}+\bar{\nu} & \\
\searrow & \\
& \bar{\nu}+e_{D}^{-} \rightarrow \bar{\nu}+e_{D}^{-}
\end{array}
$$

occurring through the intermediate propagation of an antineutrino, where $\mathrm{n}$ (neutron) and $e_{D}^{-}$(electron) are the initial particles. As already mentioned we assume that the neutron, the proton and the target electron are in stationary states. Since neutrino production and detection are localized at $\vec{x}_{P}$ and $\vec{x}_{D}$, respectively, the spinors of the initial particles and the proton can be written in position space as

$$
\begin{aligned}
\psi_{p}(x) & =\psi_{p}\left(\vec{x}-\vec{x}_{P}\right) \exp \left(-i E_{p} t\right) \\
\psi_{n}(x) & =\psi_{n}\left(\vec{x}-\vec{x}_{P}\right) \exp \left(-i E_{n} t\right) \\
\psi_{e D}(x) & =\psi_{e D}\left(\vec{x}-\vec{x}_{D}\right) \exp \left(-i E_{e D} t\right)
\end{aligned}
$$

respectively. The functions $\psi_{p, n, e D}(\vec{y})$ are peaked at $\vec{y}=0$. The final particles will be described by plane waves. 
The weak interaction Lagrangians relevant for production and detection are

$$
\begin{aligned}
\mathcal{L}_{P}(x)= & -\frac{G_{F}}{\sqrt{2}} \cos \vartheta_{c} \sum_{j} U_{e j} \bar{e}(x) \gamma^{\lambda}\left(1-\gamma_{5}\right) \nu_{j}(x) \bar{p}(x) \gamma_{\lambda}\left(1-\tilde{g} \gamma_{5}\right) n(x)+\text { h.c. }, \\
\mathcal{L}_{D}(x)= & -\frac{G_{F}}{\sqrt{2}} \sum_{j, k}\left\{\bar{e}(x) \gamma^{\mu} P_{L} e(x)\left(2 U_{e j}^{*} U_{e k}+\delta_{j k}\left(g_{V}+g_{A}\right)\right)+\right. \\
& \left.+\bar{e}(x) \gamma^{\mu} P_{R} e(x) \delta_{j k}\left(g_{V}-g_{A}\right)\right\} \bar{\nu}_{j}(x) \gamma_{\mu}\left(1-\gamma_{5}\right) \nu_{k}(x),
\end{aligned}
$$

respectively, where $G_{F}$ is the Fermi coupling constant, $\vartheta_{c}$ the Cabibbo angle, $g_{V}=$ $2 \sin ^{2} \theta_{W}-1 / 2, g_{A}=-1 / 2, \theta_{W}$ the Weinberg angle, $\tilde{g} \approx 1.26$ and $P_{R, L}=\left(1 \pm \gamma_{5}\right) / 2$. All fields occurring in eq. (5) are mass eigenfields. The amplitude for the process (3) with an antineutrino of mass $m_{k}$ in the final state is given by

$$
\mathcal{A}_{k}=\left\langle p, \bar{\nu}_{k}\left(\vec{p}_{\nu}^{\prime}\right), e^{-}\left(\vec{p}_{e}^{\prime}\right), e_{D}^{-}\left(\vec{p}_{e D}^{\prime}\right)\left|T\left[\int d^{4} x_{1} \int d^{4} x_{2} \mathcal{L}_{P}\left(x_{1}\right) \mathcal{L}_{D}\left(x_{2}\right)\right]\right| n, e_{D}^{-}\right\rangle .
$$

With the neutrino propagators of the mass eigenstate neutrinos

$$
\left\langle 0\left|T\left[\nu_{j}\left(x_{1}\right) \bar{\nu}_{j}\left(x_{2}\right)\right]\right| 0\right\rangle=i \int \frac{d^{4} q}{(2 \pi)^{4}} \frac{\not q+m_{j}}{q^{2}-m_{j}^{2}+i \epsilon} \mathrm{e}^{-i q \cdot\left(x_{1}-x_{2}\right)},
$$

we obtain the amplitude

$$
\begin{aligned}
\mathcal{A}_{k}= & \frac{G_{F}^{2} \cos \vartheta_{c}}{2} i \sum_{j} \int d^{4} x_{1} \int d^{4} x_{2} \int \frac{d^{4} q}{(2 \pi)^{4}} \mathrm{e}^{-i q \cdot\left(x_{1}-x_{2}\right)} \\
& \exp \left(-i\left(E_{n}-E_{p}\right) t_{1}-i E_{e D} t_{2}\right) \exp \left(i p_{e}^{\prime} \cdot x_{1}+i\left(p_{\nu}^{\prime}+p_{e D}^{\prime}\right) \cdot x_{2}\right) \\
& J_{\lambda}\left(\vec{x}_{1}-\vec{x}_{P}\right) \bar{u}_{e}\left(\vec{p}_{e}^{\prime}\right) \gamma^{\lambda}\left(1-\gamma_{5}\right) U_{e j}\left(\frac{\not q+m_{j}}{q^{2}-m_{j}^{2}+i \epsilon}\right) \gamma^{\mu}\left(1-\gamma_{5}\right) v_{k}\left(\vec{p}_{\nu}^{\prime}\right) \\
& \bar{u}_{e}\left(\vec{p}_{e D}^{\prime}\right) \gamma_{\mu}\left[P_{L}\left(2 U_{e j}^{*} U_{e k}+\delta_{j k}\left(g_{V}+g_{A}\right)\right)+P_{R} \delta_{j k}\left(g_{V}-g_{A}\right)\right] \psi_{e D}\left(\vec{x}_{2}-\vec{x}_{D}\right)
\end{aligned}
$$

where

$$
J_{\lambda}\left(\vec{x}_{1}-\vec{x}_{P}\right) \equiv \bar{\psi}_{p}\left(\vec{x}_{1}-\vec{x}_{P}\right) \gamma_{\lambda}\left(1-\tilde{g} \gamma_{5}\right) \psi_{n}\left(\vec{x}_{1}-\vec{x}_{P}\right) .
$$

Note that the amplitude (8) is not antisymmetric with respect to the exchange of the final electrons because the electron generated in the $\beta$-decay never reaches the detector or, in other words, it is absorbed in the reactor and thus takes part in the formation of final states orthogonal to single electron states.

The integration over $t_{1}$ and $t_{2}$ in eq. (8) can easily be carried out leading to the delta functions $\delta\left(q_{0}+E_{2}\right)$ and $\delta\left(q_{0}+E_{1}\right)$. Furthermore, we use the relation

$$
\int_{-\infty}^{\infty} d x \mathrm{e}^{-i k x} f(x+b)=\mathrm{e}^{i k b} \tilde{f}(k)
$$


where $\tilde{f}$ is the Fourier transform of $\mathrm{f}$, in the integrations over $\vec{x}_{1}$ and $\vec{x}_{2}$. Thus we obtain

$$
\begin{aligned}
\mathcal{A}_{k}= & i \frac{G^{2} \cos \vartheta_{c}}{2(2 \pi)^{2}} \mathrm{e}^{-i \vec{p}_{1} \cdot \vec{x}_{P}-i \vec{p}_{2} \cdot \vec{x}_{D}} \sum_{j} \int d^{4} q \delta\left(q_{0}+E_{1}\right) \delta\left(q_{0}+E_{2}\right) \mathrm{e}^{-i \vec{q} \cdot \vec{L}} \\
& \tilde{J}_{\lambda}\left(\vec{p}_{1}-\vec{q}\right) \bar{u}_{e}\left(\vec{p}_{1}\right) \gamma^{\lambda}\left(1-\gamma_{5}\right) U_{e j}\left(\frac{\not 1+m_{j}}{q^{2}-m_{j}^{2}+i \epsilon}\right) \gamma_{\mu}\left(1-\gamma_{5}\right) v_{k}\left(\vec{p}_{\nu}^{\prime}\right) \\
& \bar{u}_{e}\left(\vec{p}_{e D}^{\prime}\right) \gamma^{\mu}\left[P_{L}\left(2 U_{e j}^{*} U_{e k}+\delta_{j k}\left(g_{V}+g_{A}\right)\right)+P_{R} \delta_{j k}\left(g_{V}-g_{A}\right)\right] \tilde{\psi}_{e D}\left(\vec{p}_{2}+\vec{q}\right)
\end{aligned}
$$

where we have defined

$$
\begin{array}{ll}
E_{1} \equiv E_{n}-E_{p}-E_{e}^{\prime}, & \vec{p}_{1} \equiv \vec{p}_{e}^{\prime}, \\
E_{2} \equiv E_{\nu}^{\prime}+E_{e D}^{\prime}-E_{e D}, & \vec{p}_{2} \equiv \vec{p}_{e D}^{\prime}+\vec{p}_{\nu}^{\prime} .
\end{array}
$$

Now only the integration over q remains. Since our initial and final states are all energy eigenstates, the integration over $q^{0}$ leads to the usual $\delta$-function $\delta\left(E_{1}-E_{2}\right)$ expressing that the initial energy is equal to the final energy of the total process (3). The analogous $\delta$-functions corresponding to momentum are smeared out by the initial momentum distributions and therefore we have a non-trivial $d^{3} q$ integration in the amplitude. We can, however, take advantage of the fact that this integration actually amounts to calculating a Fourier transform and evaluating it at the coordinate $\vec{L}$, the macroscopic distance between neutrino production and detection (see eqs. (41)). Hence we can apply the theorem proved in the appendix (see eqs. (A.1) and (A.2)) which enables us to calculate the leading term of the amplitude for large $L$.

Let us for a moment dwell upon the mathematical requirements of this theorem in the context of the phyiscal problem under discussion. The wave functions eq. (4) represent bound states. It is therefore reasonable to assume that they fall off exponentially and consequently the Fourier transforms $\tilde{J}_{\lambda}$ and $\tilde{\psi}_{e D}$ are infinitely many times differentiable. This meets the requirement of differentiability in the theorem. It remains to discuss the behaviour of $\tilde{J}_{\lambda}$ and $\tilde{\psi}_{e D}$ for $|\vec{q}| \rightarrow \infty$. If bound states in coordinate space are also infinitely many times differentiable in addition to their exponential fall-off then their Fourier transforms have the same properties. However, bound state wave functions are in general not everywhere infinitely many times differentiable in coordinate space. A typical example is given by the ground state wave function of the hydrogen atom which is not differentiable at the origin. Its Fourier transform nevertheless decreases with the fourth power of $|\vec{q}|$ which meets the conditions for the decrease of the function in the theorem. Therefore the physics under consideration seems to comply naturally with the mathematical requirements.

Noting that the constant $A$ of the theorem corresponds to $q_{0}^{2}-m_{j}^{2}$, eq. (A.2) tells us that the asymptotic limit $L \rightarrow \infty$ of eq. (11) is given by

$$
\begin{aligned}
\mathcal{A}_{k}^{\infty}= & -i \frac{G_{F}^{2} \cos \vartheta_{c}}{4} \frac{1}{L} \delta\left(E_{1}-E_{2}\right) \mathrm{e}^{-i \vec{p}_{1} \cdot \vec{x}_{P}-i \vec{p}_{2} \cdot \vec{x}_{D}} \\
& \sum_{j} \mathrm{e}^{i q_{j} L} \tilde{J}_{\lambda}\left(\vec{p}_{1}+q_{j} \vec{l}\right) \bar{u}_{e}\left(\vec{p}_{1}\right) \gamma^{\lambda}\left(1-\gamma_{5}\right) U_{e j}\left(q_{0} \gamma^{0}+q_{j} \vec{l} \cdot \vec{\gamma}+m_{j}\right) \gamma_{\mu}\left(1-\gamma_{5}\right) v_{k}\left(\vec{p}_{\nu}^{\prime}\right) \\
& \bar{u}_{e}\left(\vec{p}_{e D}^{\prime}\right) \gamma^{\mu}\left[P_{L}\left(2 U_{e j}^{*} U_{e k}+\delta_{j k}\left(g_{V}+g_{A}\right)\right)+P_{R} \delta_{j k}\left(g_{V}-g_{A}\right)\right] \tilde{\psi}_{e D}\left(\vec{p}_{2}-q_{j} \vec{l}\right)(13)
\end{aligned}
$$


with

$$
\vec{l} \equiv \frac{\vec{L}}{|\vec{L}|}, \quad q_{0}=-E_{1}=-E_{2} \quad \text { and } \quad q_{j} \equiv \sqrt{q_{0}^{2}-m_{j}^{2}}
$$

This equation can be interpreted in such a way that $\mathcal{A}_{k}^{\infty}$ is a sum over terms which contain a real antineutrino of mass $m_{j}$. The reason is that the 4 -vector $p_{j}$ defined by

$$
p_{j}^{0} \equiv E_{\nu} \equiv-q^{0}=E_{2}=E_{e D}^{\prime}+E_{\nu}^{\prime}-E_{e D} \geq 0 \quad \text { and } \quad \vec{p}_{j} \equiv q_{j} \vec{l}
$$

can be identified as the 4-momentum of this antineutrino. The spatial part of $p_{j}$ correctly points from $\vec{x}_{P}$ to $\vec{x}_{D}$. Furthermore, with a suitable renormalization of the 4 -spinors $v$ the identity

$$
q_{0} \gamma^{0}+q_{j} \vec{l} \cdot \vec{\gamma}+m_{j}=-\not p_{j}+m_{j}=-\sum_{ \pm s} v_{j}\left(\vec{p}_{j}, s\right) \bar{v}_{j}\left(\vec{p}_{j}, s\right)
$$

shows that apart from the factors $1 / L$ and $\exp \left(i q_{j} L\right)$ the amplitude $\mathcal{A}_{k}^{\infty}$ is just the sum over the products of production and detection amplitude of antineutrinos with mass $m_{j}$.

\section{Oscillating amplitude and cross section}

We expect that under certain conditions the cross section for the reaction (3) $\left(n+e_{D}^{-} \rightarrow\right.$ $\left.e^{-}+e_{D}^{-}+\bar{\nu}\right)$ exhibits an oscillatory dependence on the distance $L$. Considering first the asymptotic amplitude $\mathcal{A}_{k}^{\infty}$ and assuming that $E_{\nu} \gg m_{j} \forall j$ we can make the following remarks and observations to this effect. (Most of them have already been discussed in the context of the quantum-mechanical approach of ref. [3].)

- In order to obtain $\mathcal{A}_{j}^{\infty}$ we have performed the asymptotic limit $L \rightarrow \infty$. Looking at theorem proved in the appendix it is evident that " $L$ large" means $\bar{E}_{\nu} L / \hbar c \approx$ $\bar{E}_{\nu} L / 2 \cdot 10^{-13} \mathrm{MeV} \cdot \mathrm{m} \gg 1$ where $\bar{E}_{\nu}$ is an average antineutrino energy and $L$ is measured in meters. For every thinkable neutrino experiment this is very well fulfilled and corrections to $\mathcal{A}_{k}^{\infty}$ are suppressed by $\left(\bar{E}_{\nu} L / \hbar c\right)^{-1 / 2}$.

- The factor $1 / L$ in the asymptotic amplitude corresponds to the geometrical decrease of the neutrino flux by $1 / L^{2}$ in the cross section.

- Looking at eq. (13) we conclude that neutrino oscillations with masses $m_{j}, m_{k}$ can only take place if

$$
\left|q_{j}-q_{k}\right| \lesssim \sigma_{J} \quad \text { and } \quad\left|q_{j}-q_{k}\right| \lesssim \sigma_{D}
$$

where $\sigma_{J}$ and $\sigma_{D}$ are the widths of the functions $\tilde{J}$ and $\tilde{\psi}_{e D}$, respectively. In coordinate space this simply means that the widths of $J$ and $\psi_{e D}$ must both be smaller than the oscillation length $l_{\text {osc }}=4 \pi E_{\nu} /\left|m_{j}^{2}-m_{k}^{2}\right| \approx 2.48 \mathrm{~m} \cdot E_{\nu}(\mathrm{MeV}) / \Delta m^{2}\left(\mathrm{eV}^{2}\right)$.

- To evaluate this condition for reactor neutrinos we note that $\hbar c / \sigma_{J} \gtrsim 10^{-15} \mathrm{~m}$ and $\hbar c / \sigma_{D} \gtrsim 10^{-10} \mathrm{~m}$. From the latter estimate one gets the condition $\Delta m^{2} \lesssim(100 \mathrm{keV})^{2}$ 3. 
- The coherence length is infinite in our calculation since all initial and final states are stationary.

- Our calculation was based on the assumption that neutrinos are of Dirac nature. In the case of Majorana neutrinos the diagonal vector currents in $\mathcal{L}_{D}$ eq. (5) are identically zero. As a consequence, in $\mathcal{A}_{k}$ eq. (\$) the second $1-\gamma_{5}$ has to be replaced by $-2 \gamma_{5}$ for $j=k$. This introduces the well known difference of order $m_{j} / E_{\nu}$ between the amplitudes of Majorana and Dirac neutrinos [2].

Finally we come to the cross section or event rate in order to make contact with the usual oscillation picture. If $\left|q_{j}-q_{k}\right| \ll \min \left(\sigma_{J}, \sigma_{D}\right)$ is fulfilled $\forall j, k$ in addition to the assumption that all neutrinos are ultrarelativistic, then we can take the limit $m_{j} \rightarrow 0 \forall j$ in all terms of $\mathcal{A}_{k}^{\infty}$ except the exponential factors $\exp \left(i q_{j} L\right)$. Then calculating the cross section one would arrive at the same result as can be obtained by the following heuristic consideration: The probability to find a neutrino $\bar{\nu}_{\ell}$ at a distance $L$ from the source is given by $P_{\ell}=\left|\sum_{j} U_{e j} U_{\ell j}^{*} \mathrm{e}^{i q_{j} L}\right|^{2}$. Thus the number of events in elastic $\bar{\nu} e^{-}$ scattering at a certain neutrino energy $E_{\nu}$ is proportional to $\sum_{\ell} P_{\ell} \sigma\left(\bar{\nu}_{\ell} e^{-} ; E_{\nu}\right)$ where the $\sigma\left(\bar{\nu}_{\ell} e^{-} ; E_{\nu}\right)(\ell=e, \mu, \tau)$ are the elastic scattering cross sections as given by the SM. Note that $E_{\nu}$ can be reconstructed via

$$
E_{\nu}=m_{e}\left(\sqrt{1+\frac{2 m_{e}}{T}} \cos \alpha-1\right)^{-1}
$$

where $T=E_{e D}^{\prime}-m_{e}$ and $\alpha$ is the angle between $\vec{L}$ and $\vec{p}_{e D}^{\prime}$, the momentum of the recoil electron. Thus the event rate is given by

$$
\frac{d N}{d E_{\nu}}=N_{0}\left\{\left.\left.\left|\sum_{j}\right| U_{e j}\right|^{2} \mathrm{e}^{i q_{j} L}\right|^{2}\left(\sigma\left(\bar{\nu}_{e} e^{-} ; E_{\nu}\right)-\sigma\left(\bar{\nu}_{\mu} e^{-} ; E_{\nu}\right)\right)+\sigma\left(\bar{\nu}_{\mu} e^{-} ; E_{\nu}\right)\right\}
$$

with a normalization constant $N_{0}$.

In conclusion we want to stress once more the central importance of the theorem demonstrated in the appendix. It is not only relevant in the context discussed here but it shows in general under which circumstances the total amplitude for particle production and its subsequent scattering factorizes into a product (or sum of products) of production amplitude and scattering amplitude. In the case of neutrino oscillations it leads to a transparent and simple field-theoretical treatment without ambiguities or conceptional problems [3]: all neutrino states or fields are mass eigenstates or eigenfields and the neutrino "wave packet" is totally determined by the production mechanism. The method described here also provides the correct answer in cases where the standard approach to neutrino oscillations fails. We want to mention, however, that some more work has to be done to extend our method because it is not straightforwardly transferable to the case of accelerator neutrinos and it has the deficiency of an unrealistic infinite coherence length for neutrino oscillations. 


\section{Appendix}

Theorem: Let $\Phi: \mathbf{R}^{3} \rightarrow \mathbf{R}^{3}$ be a three times continuously differentiable function such that $\Phi$ itself and all its first and second derivatives decrease at least like $1 / \vec{q}^{2}$ for $|\vec{q}| \rightarrow \infty$, $A$ a real number and

$$
J(\vec{L}) \equiv \int d^{3} q \Phi(\vec{q}) \mathrm{e}^{-i \vec{q} \cdot \vec{L}} \frac{1}{A-\vec{q}^{2}+i \epsilon} .
$$

Then in the asymptotic limit $L=|\vec{L}| \rightarrow \infty$ one obtains for $A>0$

$$
J(\vec{L})=-\frac{2 \pi^{2}}{L} \Phi(-\sqrt{A} \vec{L} / L) \mathrm{e}^{i \sqrt{A} L}+\mathcal{O}\left(L^{-3 / 2}\right)
$$

whereas for $A<0$ the integral decreases like $L^{-2}$.

Remark: In order to make the proof of this theorem transparent we will first introduce three lemmas and then divide the proof into several steps.

Lemma 1: Let $f$ be a three times continuously differentiable function and

$$
I(r) \equiv \int_{0}^{\pi} d \theta \sin \theta \mathrm{e}^{-i r \cos \theta} f(\theta) .
$$

Then in the asymptotic limit $r \rightarrow \infty$ one obtains

$$
I(r)=-\frac{\mathrm{e}^{-i r} f(0)-\mathrm{e}^{i r} f(\pi)}{i r}+\mathcal{O}\left(r^{-3 / 2}\right) .
$$

Proof: We first perform a partial integration resulting in

$$
I(r)=-\frac{\mathrm{e}^{-i r} f(0)-\mathrm{e}^{i r} f(\pi)}{i r}-\frac{1}{i r} I_{1}(r)
$$

with

$$
I_{1}(r)=\int_{0}^{\pi} d \theta \mathrm{e}^{-i r \cos \theta} f^{\prime}(\theta)
$$

It is convenient to split $I_{1}(r)$ into the two parts

$$
I_{1}(r)=\int_{0}^{\pi / 2} d \theta \mathrm{e}^{-i r \cos \theta} f^{\prime}(\theta)+\int_{0}^{\pi / 2} d \theta \mathrm{e}^{i r \cos \theta} f^{\prime}(\pi-\theta) .
$$

In the following we only discuss the first integral in this equation since the second one is treated analogously. Defining $g(\theta)=\left(f^{\prime}(\theta)-f^{\prime}(0)\right) / \sin \theta$ we obtain

$$
\int_{0}^{\pi / 2} d \theta \mathrm{e}^{-i r \cos \theta} f^{\prime}(\theta)=\int_{0}^{\pi / 2} d \theta \mathrm{e}^{-i r \cos \theta} f^{\prime}(0)+\int_{0}^{\pi / 2} d \theta \sin \theta \mathrm{e}^{-i r \cos \theta} g(\theta) .
$$

It is easy to show that $g$ is twice continuously differentiable in the intervall $[0, \pi / 2]$ as a consequence of the three times continuous differentiability of $f$. Therefore one can apply a partial integration to the second integral on the right-hand side of eq. (A.8) in the same 
way as to eq. (A.3) and thus this integral decreases like $1 / r$ in the asymptotic limit (see eq. A.5). Together with the $1 / r$ in front of $I_{1}(r)$ in eq. (A.5) there is an overall decrease of $1 / r^{2}$. Therefore it remains to consider the asymptotic behaviour of

$$
\int_{0}^{\pi / 2} d \theta \mathrm{e}^{-i r \cos \theta}=\frac{1}{2}\left(\int_{0}^{1} d z \frac{\mathrm{e}^{-i r z}}{\sqrt{1-z^{2}}}-\int_{\pi / r}^{1+\pi / r} d z \frac{\mathrm{e}^{-i r z}}{\sqrt{1-\left(z-\frac{\pi}{r}\right)^{2}}}\right) .
$$

The right-hand side of this equation allows to deduce the upper bound

$$
\begin{aligned}
& \mid \int_{0}^{\pi / 2} d \theta \mathrm{e}^{-i r \cos \theta \mid \leq} \\
& \quad \leq \frac{1}{2}\left[\int_{0}^{\pi / r} d z \frac{1}{\sqrt{1-z^{2}}}+\int_{1}^{1+\pi / r} d z \frac{1}{\sqrt{1-\left(z-\frac{\pi}{r}\right)^{2}}}+\right. \\
& \left.\quad+\int_{\pi / r}^{1} d z\left(\frac{1}{\sqrt{1-z^{2}}}-\frac{1}{\sqrt{1-\left(z-\frac{\pi}{r}\right)^{2}}}\right)\right]= \\
& \quad=\arcsin \sqrt{\frac{2 \pi}{r}-\frac{\pi^{2}}{r^{2}}} .
\end{aligned}
$$

Thus the integral of eq. (A.9) is bounded by a function with asymptotic behaviour $\mathcal{O}(1 / \sqrt{r})$ and lemma 1 follows.

Lemma 2: Let $f$ be a three times continuously differentiable function and $w$ be a real number with $|w|<1$. Then the integral

$$
I_{w}(r) \equiv \int_{0}^{\pi} d \theta \sin \theta \mathrm{e}^{-i r \cos \theta} \sin (w r \cos \theta) f(\theta)
$$

has the asymptotic behaviour

$$
I_{w}(r)=\frac{\mathrm{e}^{-i r}}{r} f(0) \frac{w \cos w r+i \sin w r}{1-w^{2}}-\frac{\mathrm{e}^{i r}}{r} f(\pi) \frac{w \cos w r-i \sin w r}{1-w^{2}}+\mathcal{O}\left(r^{-3 / 2}\right)
$$

in the limit $r \rightarrow \infty$.

Proof: The proof of this lemma follows from lemma 1 by using $\sin \alpha=\left(\mathrm{e}^{i \alpha}-\mathrm{e}^{-i \alpha}\right) / 2 i$ for $\alpha=w r \cos \theta$.

Lemma 3: Let $\psi: \mathbf{R}^{3} \rightarrow \mathbf{R}^{3}$ be a twice continuously differentiable function such that the function itself and its first and second derivatives are absolutely integrable. Then the integral

$$
I(\vec{q}) \equiv \int d^{3} q \psi(\vec{q}) \mathrm{e}^{-i \vec{q} \cdot \vec{L}}
$$

decreases like $L^{-2}$ for $L \rightarrow \infty$. 
Proof: According to the assumption, $\triangle \psi$ is absolutely integrable and with a twofold partial integration we obtain

$$
\int d^{3} q(\triangle \psi)(\vec{q}) \mathrm{e}^{-i \vec{q} \cdot \vec{L}}=-\vec{L}^{2} \int d^{3} q \psi(\vec{q}) \mathrm{e}^{-i \vec{q} \cdot \vec{L}}
$$

which proves lemma 3 .

Proof of the theorem: The main point in the proof is to meticulously take care to perform the limit $\epsilon \rightarrow 0$ before investigating the asymptotic limit $L \rightarrow \infty$.

Step 1: For $A<0$ we apply lemma 3 and thus obtain the second part of the theorem. In the following $A$ will always be positive.

Step 2: $\quad$ Next we split $J(\vec{L})$ into the integrals

$$
J_{1}(\vec{L})=-\frac{i \pi}{2} \sqrt{A} \int_{S^{2}} d \Omega \Phi(\sqrt{A} \vec{n}) \mathrm{e}^{-i \sqrt{A} \vec{n} \cdot \vec{L}}
$$

originating in the $\delta$-function of the limit $\epsilon \rightarrow 0$ and

$$
J_{2}(\vec{L})=-\int d^{3} q \Phi(\vec{q}) \mathrm{e}^{-i \vec{q} \cdot \vec{L}} \frac{q^{2}-A}{\left(q^{2}-A\right)^{2}+\epsilon^{2}}
$$

which represents the principal value of this limit. $S^{2}$ denotes the 2-dimensional unit sphere, $q \equiv|\vec{q}|$ and $\vec{n} \equiv \vec{q} / q$.

Step 3: We choose two numbers $\delta$ and $\eta$ such that $0<\delta<\eta<\sqrt{A}$ and a symmetric $C^{\infty}$ function $h$ such that $0 \leq h(v) \leq 1$ for all $v \in \mathbf{R}, h(v)=1$ for $|v| \leq \delta$ and $h(v)=0$ for $|v| \geq \eta$. Then $J_{2}=\sum_{k=1}^{3} J_{2 k}$ with

$$
\begin{aligned}
J_{21}(\vec{L}) \equiv & -\int d^{3} q \mathrm{e}^{-i \vec{q} \cdot \vec{L}}(\Phi(\vec{q})-\Phi(\sqrt{A} \vec{n}) h(q-\sqrt{A})) \frac{q^{2}-A}{\left(q^{2}-A\right)^{2}+\epsilon^{2}}, \\
J_{22}(\vec{L}) \equiv & -\int_{S^{2}} d \Omega \int_{0}^{\infty} d q \mathrm{e}^{-i \vec{q} \cdot \vec{L}} \Phi(\sqrt{A} \vec{n}) h(q-\sqrt{A}) \cdot \\
& \cdot\left(\frac{q^{2}\left(q^{2}-A\right)}{\left(q^{2}-A\right)^{2}+\epsilon^{2}}-\frac{2 A^{3 / 2}(q-\sqrt{A})}{4 A(q-\sqrt{A})^{2}+\epsilon^{2}}\right) \\
J_{23}(\vec{L}) \equiv & -\int_{S^{2}} d \Omega \int_{0}^{\infty} d q \mathrm{e}^{-i \vec{q} \cdot \vec{L}} \Phi(\sqrt{A} \vec{n}) h(q-\sqrt{A}) \frac{2 A^{3 / 2}(q-\sqrt{A})}{4 A(q-\sqrt{A})^{2}+\epsilon^{2}} .
\end{aligned}
$$

In these three integrals the limit $\epsilon \rightarrow 0$ can be performed. In $J_{21}$ one obtains an integrand which is twice continuously differentiable and therefore $J_{21}$ decreases like $L^{-2}$ for $L \rightarrow \infty$ according to lemma 3. The function within the parentheses of $J_{22}$ gives $(q+\sqrt{A} / 2) /(q+$ $\sqrt{A}$ ) in the limit $\epsilon \rightarrow 0$ and with the properties of $h$ the same asymptotic behaviour as that of $J_{21}$ results. Finally, with the transformation $v=q-\sqrt{A}$ the integral $J_{23}$ can be cast into the form

$$
J_{23}(\vec{L})=\frac{i}{2} \sqrt{A} \int_{S^{2}} d \Omega \Phi(\sqrt{A} \vec{n}) \mathrm{e}^{-i \sqrt{A} \vec{n} \cdot \vec{L}} \int_{-\eta}^{\eta} d v h(v) \frac{\sin (v \vec{n} \cdot \vec{L})}{v} .
$$


Step 4: To perform the integration over $S^{2}$ we take

$$
\vec{L}=L\left(\begin{array}{l}
0 \\
0 \\
1
\end{array}\right) \equiv L \vec{e}_{z} \quad \text { and } \quad \vec{n}(\theta, \phi)=\left(\begin{array}{c}
\sin \theta \cos \phi \\
\sin \theta \sin \phi \\
\cos \theta
\end{array}\right)
$$

Then the application of lemma 1 with $r=\sqrt{A} L$ shows that

$$
J_{1}(\vec{L})=\frac{\pi^{2}}{L}\left(\mathrm{e}^{-i \sqrt{A} L} \Phi\left(\sqrt{A} \vec{e}_{z}\right)-\mathrm{e}^{i \sqrt{A} L} \Phi\left(-\sqrt{A} \vec{e}_{z}\right)\right)+\mathcal{O}\left(L^{-3 / 2}\right) .
$$

Step 5: By virtue of lemma 2 and taking $w=v / \sqrt{A}$, the asymptotic behaviour of $J_{23}$ is given by

$$
\begin{aligned}
J_{23}(\vec{L})= & \frac{i \pi}{L} \int_{-\eta}^{\eta} d v \frac{h(v)}{v\left(1-\frac{v^{2}}{A}\right)}\left[\mathrm{e}^{-i \sqrt{A} L} \Phi\left(\sqrt{A} \vec{e}_{z}\right)\left(\frac{v}{\sqrt{A}} \cos (v L)+i \sin (v L)\right)-\right. \\
& \left.-\mathrm{e}^{i \sqrt{A} L} \Phi\left(-\sqrt{A} \vec{e}_{z}\right)\left(\frac{v}{\sqrt{A}} \cos (v L)-i \sin (v L)\right)\right]+\mathcal{O}\left(L^{-3 / 2}\right) .
\end{aligned}
$$

The terms with $\cos (v L)$ decrease faster than any power of $1 / L$ by a corollary of lemma 3 whereas the $\delta$-function property of $\sin (v L) / \pi v$ leads to

$$
J_{23}(\vec{L})=-\frac{\pi^{2}}{L}\left(\mathrm{e}^{-i \sqrt{A} L} \Phi\left(\sqrt{A} \vec{e}_{z}\right)+\mathrm{e}^{i \sqrt{A} L} \Phi\left(-\sqrt{A} \vec{e}_{z}\right)\right)+\mathcal{O}\left(L^{-3 / 2}\right) .
$$

The correction to the $\delta$-function is proportional to

$$
\int_{-\infty}^{\infty} d v\left(\frac{h(v)}{1-\frac{v^{2}}{A}}-1\right) \frac{\sin (v L)}{v}
$$

and therefore decreases faster than any power of $1 / L$ as can easily be seen by repeated partial integration. Finally, the theorem is obtained by summing the results of steps 4 and 5 . 


\section{References}

[1] B. Pontecorvo, Sov. Phys. JETP 26, 984 (1968).

[2] S. M. Bilenky and S. T. Petcov, Rev. Mod. Phys. 59, 671 (1987);

B. Kayser, F. Gibrat-Debu and F. Perrier, The Physics of Massive Neutrinos (World Scientific, Singapore 1989);

R. N. Mohapatra and P. B. Pal, Massive Neutrinos in Physics and Astrophysics (World Scientific, Singapore, 1991);

C. W. Kim and A. Pevsner, Neutrinos in Physics and Astrophysics (harwood academic publishers, Chur, 1993).

[3] J. Rich, Phys. Rev. D 48, 4318 (1993).

[4] Boris Kayser, Phys. Rev. D 24, 110 (1981).

[5] C. Giunti, C. W. Kim, J. A. Lee and U. W. Lee, Phys. Rev. D 48, 4310 (1993).

[6] J. Rich, D. Lloyd Owen and M. Spiro, Phys. Rep. 151, 267 (1987);

Boris Kayser, in Neutrino 90, Proceedings of the 14th International Conference on Neutrino Physics and Astrophysics, Geneva, Switzerland, 1990, edited by J. Panam and K. Winter [Nucl. Phys. B(proc. Suppl.) 19, 177 (1991)];

C. Giunti, C. W. Kim and U. W. Lee, Phys. Rev. D 45, 2414 (1992).

[7] C. Broggini (MUNU Collaboration), in Perspectives in Neutrinos, Atomic Physics and Gravitation, Proceedings of the XIIIth Moriond Workshop, Villar sur Ollon, Switzerland, 1993, edited by J. Trân Thanh Vân, T. Damour, E. Hinds and J. Wilkerson. 